\title{
COMPUTATION OF CONDUCTANCE AND CAPACITANCE FOR IC INTERCONNECTS ON A GENERAL LOSSY MULTILAYER SUBSTRATE
}

\author{
H. YMERI ${ }^{\mathrm{a}, *}$, B. NAUWELAERS ${ }^{\mathrm{a}}$ and KAREN MAEX ${ }^{\mathrm{a}, \mathrm{b}}$ \\ ${ }^{a}$ Katholieke Universiteit Leuven, Department of Electrical Engineering \\ (ESAT), Division ESAT-TELEMIC, Kasteelpark Arenberg 10, \\ B-3001 Leuven-Heverlee, Belgium; ${ }^{b} I M E C$, Kapeldreef 75, \\ B-3001 Leuven, Belgium
}

(Received 6 February 2001; In final form 19 March 2001)

In this paper a simple method for analysis and modelling of transmission interconnect lines on general lossy multilayer substrates at high bit rates is presented. The analysis is based on semi-analytical Green's function approach and recurrence relation between the coefficients of potential in $n$ and $n+1$ layers, respectively. The electromagnetic concept of free charge density is applied. It allows us to obtain integral equations between electric scalar potential and charge density distributions. These equations are solved by the Galerkin procedure of the Method of Moments. New approach is especially adequate to model 2-D layered structures with planar boundaries for frequencies up to $20 \mathrm{GHz}$ (quasistationary field approach). The transmission line parameters (capacitance and conductance per unit length) for the given interconnect multilayer geometry are computed. A discussion of the calculated line admittance in terms of technological and geometrical parameters of the structure is given. A comparison of the numerical results from the new procedure with the techniques presented in the previous publications are provided, too.

Keywords: Interconnect transmission lines; Admittance per unit length; Galerkin procedure; Lossy multilayer medium

\footnotetext{
*Corresponding author. Tel.: + 32 (16) 3218 76, Fax: + 32 (16) 3219 86, e-mail: hassan.ymeri@esat.kuleuven.ac.be
} 


\section{INTRODUCTION}

In the past few years, we have witnessed a spectacular development in the complexity and speed of operation of IC circuits. With increasing chip areas and clock rates the performance of VLSI circuits based on MIS (metal-insulator-semiconductor) technology depends more and more upon the properties of interconnections. To optimize electrical properties of IC interconnects, the estimation of the transmission line parameters requires an accurate and frequency-dependent model which includes the effects of semiconductor substrates at high frequencies. Most high-performance digital circuits are simulated before construction. In reality, we can convert theoretical results into useful CAD tools for modelling the electromagnetic behaviour of complex 3D interconnects, and transfer the field solutions into circuit parameters.

Transmission interconnect lines on MIS structures have been investigated for many years. In $[2,3]$ Hasegawa et al., presented an analysis of microstrip line on a $\mathrm{Si}-\mathrm{SiO}_{2}$ system using parallel-plate waveguide model. In [1], the new model is developed to represent fine line and wide line interconnect behaviour over a $20 \mathrm{GHz}$ frequency range and includes the substrate conductance effects. In [4], propagation properties of multilayer coplanar lines on different types of silicon substrates are investigated. In [5,6], quasi-analytical analysis of broadband properties of multiconductor transmission lines on semiconducting substrates is done, and the calculated results for line parameters as function of frequency are discussed. Numerous electromagnetic approaches have been published which contain results of numerical fullwave or quasi-TEM analyses [7-12,22-28]. We can mention, the method based upon the classical mode-matching procedure [7], the spectral-domain analysis method [8-10], and the finite element method [11] have been investigated for this structure. It has been well established that the resistivity-frequency plane can be divided into three main regions: the dissipative dielectric region, the slow-wave region, and the skin-effect region. Recently quasi-TEM analysis on coplanar structure has made the incorporation of metallic conductor losses in the analysis possible and has provided a physical basis for the construction of equivalent circuits [12]. In [29], the CAD-oriented equivalent-circuit modeling procedure based on a quasi-stationary spectral domain approach which takes into account the skin effect in the silicon 
semiconducting substrate is presented. The silicon interconnects represent an important class of lossy and dispersive transmission line structures and, therefore, should be described by frequency-dependent admitance and impedance per unit length $Y(\omega)$ and $Z(\omega)$. For more accurate parameter calculations and practical application in advanced VLSI technology, the following specific electrical properties of transmission lines on semiconductor substrate, as used in common VLSI chips, have to be considered (that are different from microwave transmission line): first, the interconnects in IC's have line widths of order of micrometers. With decreasing line width, the effects of the stripline metal loss increase. Second, in most IC interconnect structures the return lines (grounded) are placed in the same plane as the signal line and distances between signal and grounded lines are often very large, the structure cannot be treated as coplanar line. Third, for practical calculations of the parameters of the given structure full-wave procedures developed in classical electromagnetic theory and microwaves are very complicated and generaly time consuming.

In this paper, we present a new semi-analytical procedure for single and coupled interconnects on lossy silicon substrate, which accurately models the conductance and capacitance of the interconnect structure. The modeling technique is based on quasi-stationary spatial domain multilayer Green's function approach and recurrence relation between the potential coefficients of the $n$ and $n+1$ layers, respectively. The formulation of lossy coupled multiconductor interconnection lines is obtained using a multilayer dielectric Green's function in conjunction with free charge distribution on the conductor lines [14-16]. The solution is derived by applying the method of moments [17] and boundary integral equation approach. Solutions based on the boundary integral equation approach seem to be very well suited for many practical cases (discretization of only conductor interconnect bounds) - specifically for application in microelectronic interconnect structures - if the goal is to get high accuracy with low computational cost. The analytical formulas published so far do not satisfactorily describe the broadband behaviour of coupled line systems, since they are either only valid for single lines, for wide lines or in a limited frequency range. For this purpose the interconnect transmission line structures shown in Figures 1 and 2 have been taken as a basis for the following. Typical on-chip interconnects used in mixed-signal 
$\mathrm{SiO}_{2}$

\section{Silicon}

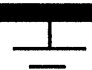

FIGURE 1 Typical on-chip interconnects used in mixed-signal intergrated circuits with ground plane.

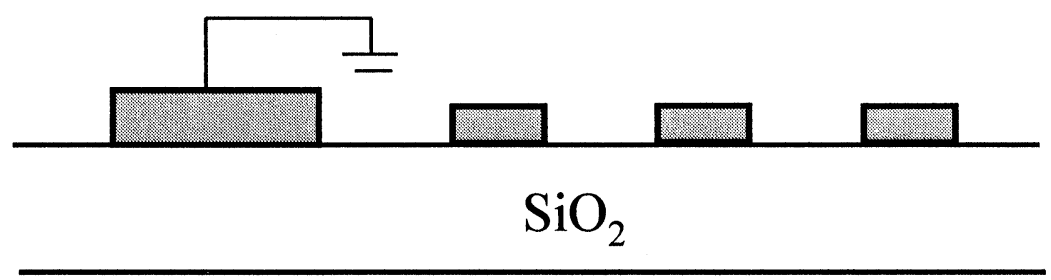

\section{Silicon}

FIGURE 2 Typical coplanar interconnects without ground plane.

integrated circuits with ground plane (Fig. 1) or coplanar interconnects without ground plane (Fig. 2), respectively.

Based on the assumption that the fields have the quasi-stationary character, a new semi-analytical approach is developed for calculation of the complex distributed line admittance per unit length (capacitance and conductance per unit length) of the VLSI interconnects. 


\section{QUASI-STATIONARY FIELD APPROACH}

In IC technology, the separation between the lines is about a few micrometers and the distance between the conductors and the ground plane is about several hundred micrometers; therefore, the coupling coefficients are very important.

Let us consider an arbitrary number $\mathrm{N}$ of metallic strips which are embedded in the top layer of a medium which consists of $\mathrm{L}$ dielectric layers, as shown in Figure 3a. The permittivity of the dielectric layers is $\varepsilon_{l}=\varepsilon_{0} \varepsilon_{r l}(1=1, \ldots, \mathrm{L})$ where $\varepsilon_{0}$ is the permittivity of free space and $\varepsilon_{r l}$ is the relative permittivity of the dielectric layers. The structure is uniform in $x$ and $y$ direction. The whole structure is bound by a rectangular box defined by planes $x=0, x=a, z=0$ and $z=d_{L}$. The $z=0$ plane is a perfectly conducting ground plane and the sidewalls

(a)
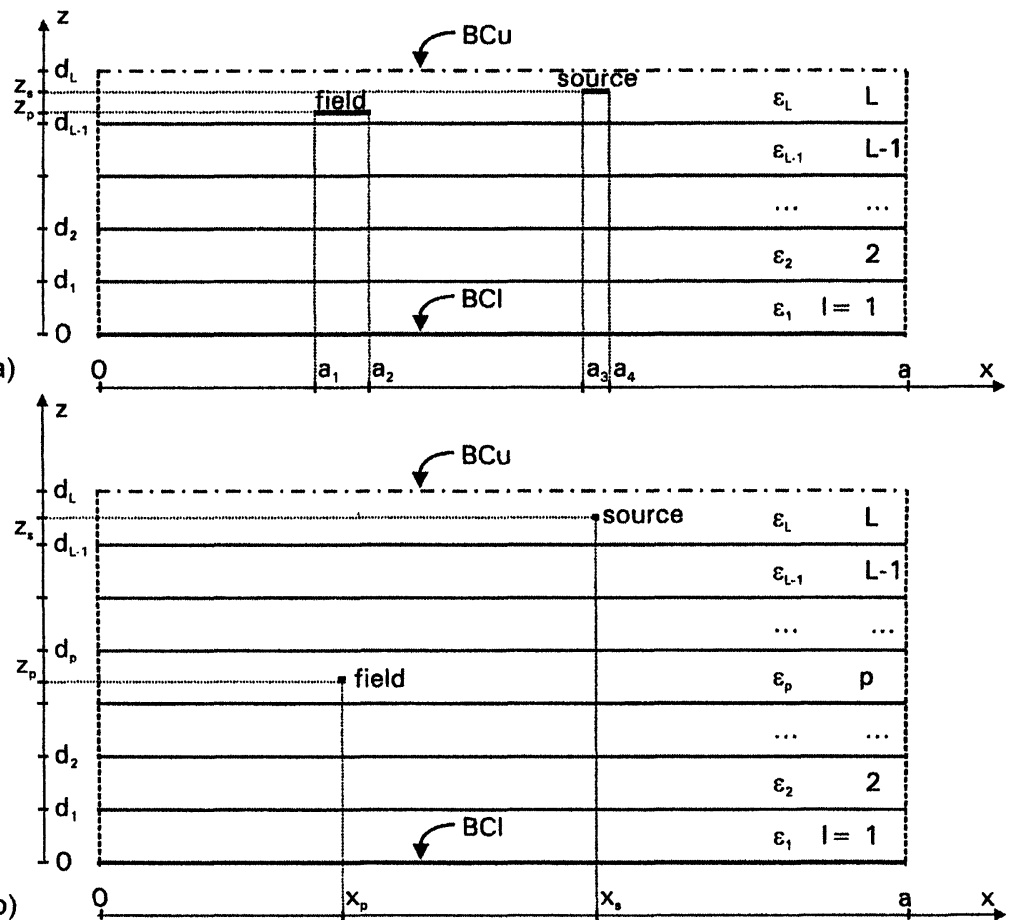

FIGURE 3 Geometry of a multilayer structure (a) with conductors in the top layer (b) for Green's function calculation. 
and the top surface are open boundaries (Neuman type boundary conditions).

If the conductivity is small enough or the frequency is high enough but still well below the quasi-stationary frequency limit, the conduction current density produced by the quasi-static field strength $\mathbf{E}$ is negligible compared to the quasi-static displacement current density $\mathrm{j} \omega \mathbf{D}$. Along with $\sigma \mathbf{E}(\sigma \ll \omega \varepsilon)$, the conduction current density $\sigma \mathbf{E}_{\mathrm{i}}$ produced by the induced field strength $\mathbf{E}_{\mathbf{i}}$ is also negligible since the fact that $\sigma \ll \omega \varepsilon\left(\sigma \mathbf{E}_{\mathbf{i}} \ll j \omega \varepsilon \mathbf{E}_{\mathbf{i}}\right)$. The contribution of $j \omega \varepsilon \mathbf{E}_{\mathbf{i}}$ is already neglected in the quasi-stationary approximation because of $\left\|j \omega \varepsilon \mathbf{E}_{\mathbf{i}}\right\| \ll$ $\left\|\mathbf{J}^{\mathbf{s}}+\mathrm{j} \omega \varepsilon \mathbf{E}\right\|$.

Hence, the field equations for the quasi-stationary approximation are

$$
\begin{aligned}
\nabla \times \mathbf{E} & =0 \\
\nabla \bullet \mathbf{D} & =\rho \\
\mathbf{D} & =\varepsilon_{0} \varepsilon_{r} \mathbf{E}
\end{aligned}
$$

just as they are in electrostatics. Thus, with known charge density distribution, the quasi-static electric field can be calculated independently of the magnetic field. Relation (1) implies that the field $\mathbf{E}$ can be written as the gradient of a potential function $\varphi$, i.e.,

$$
\mathbf{E}=-\nabla \varphi
$$

Bearing in mind the above mentioned equations, the Poisson equation for potential function can be viewed as

$$
\nabla^{2} \varphi=-\rho / \varepsilon
$$

Therefore, we present in this work a generalized approach for the calculation of the transmission line parameters for a specific geometry using quasi-stationary mode propagation (in this case equations for E-field looks as they are in quasi-TEM approximation). Since a static analysis would be valid for low frequencies only, a number of frequency dependent effects that occur at high frequencies have been included, like frequency dependencies of both the capacitances and conductances $(Y(\omega)=G+j \omega C)$ per unit length. This approach ensures that the analysis is valid up to the frequency where non-quasistationary modes can propagates in the interconnection lines, i.e., the 
cutoff frequency for the surface wave mode. It has been shown that for a $450 \mu \mathrm{m}$ thick silicon substrate wafer with $10 \Omega \mathrm{cm}$ resistivity, the cutoff frequency is about $50 \mathrm{GHz}$ [20].

The characteristic impedance matrix $\left[\mathrm{Z}_{0}\right]$ and propagation constant matrix $[\gamma]$ of interconnect multilines in a layered medium can be found as

$$
\begin{gathered}
{\left[Z_{0}\right]=\left[\begin{array}{ccc}
Z_{01} & & 0 \\
& \ddots & \\
0 & & Z_{0 N}
\end{array}\right]=[\gamma]\left([Y]\left[E_{v}\right]\right)^{-1}\left[E_{i}\right]} \\
{[\gamma]=\left[\begin{array}{lll}
\gamma_{1} & & 0 \\
& \ddots & \\
0 & & \gamma_{N}
\end{array}\right]=[\lambda]^{1 / 2} .}
\end{gathered}
$$

Equations (4) and (5) are identical to those derived from the electrical circuit theory description of a transmission line with distributed shunt admittance $[Y]=[G]+j \omega[C]$ and series impedance $[Z]=[R]+j \omega[L]$ $\left[E_{v}\right]$ and $\left[E_{i}\right]$ are the matrices of the eigenvectors of $[\mathrm{Z}][\mathrm{Y}]$ and $[\mathrm{Y}][\mathrm{Z}]$, respectively, and $[\lambda]$ contains the eigenvalues of $[\mathrm{Z}][\mathrm{Y}]$. These quantities also appear in the conventional transmission line equations satisfied by $v$ ] and $i]$ :

$$
\begin{aligned}
& \left.\frac{d v]}{d z}=-([R]+j \omega[L]) i\right] \\
& \left.\frac{d i]}{d z}=-([G]+j \omega[C]) v\right] .
\end{aligned}
$$

Here vectors $v]=\left[v_{1}, v_{2}, \ldots, v_{N}\right]^{T}$ and $\left.i\right]=\left[i_{1}, i_{2}, \ldots, i_{N}\right]^{T}$ represent voltages and currents on the interconnect lines. We have used a transmission line analogy by defining real equivalent circuit parameter matrices $[\mathrm{C}],[\mathrm{L}],[\mathrm{G}]$ and $[\mathrm{R}]$, analogous to the shunt capacitance, series inductance, shunt conductance, and series resistance per unit length matrices, of the conventional transmission line theorey. The elements of these matrices represent the equivalent self and mutual parameters of the interconnections. Only a quasi-static calculation procedure for the $[\mathrm{C}]$ matrix is, however, necessarily to find the 
following three matrices $[\mathrm{L}],[\mathrm{G}]$ and $[\mathrm{R}]$ :

- Since the inductance matrix per unit length is invariant with respect to the dielectric materials of the system of interconnect multilines, it can be calculated as

$$
[L]=\varepsilon_{0} \mu_{0}\left[C_{0}\right]^{-1}=\frac{1}{c_{0}^{2}}\left[C_{0}\right]^{-1}
$$

- where $\varepsilon_{0}$ and $\mu_{0}$ are constitutive parameters of the free space and $c_{0}$ is the speed of light. $\left[\mathrm{C}_{0}\right]^{-1}$ denotes the inverse of the capacitance matrix per unit length of the system of interconnect multilines whose dielectric materials are replaced by free space.

- The [G] matrix can be found from the complex capacitance calculation $\left(\left[\mathrm{C}^{\prime}\right]=[\mathrm{C}]-\mathrm{j}([\mathrm{G}] / \omega)\right)$, which is the capacitance calculation when the complex permittivity for the lossy dielectrics is used: $\varepsilon_{\mathrm{rc}}=\varepsilon_{\mathrm{r}}-\mathrm{j}\left(\sigma / \omega \varepsilon_{0}\right)=\varepsilon_{\mathrm{r}}(1-\mathrm{j} \tan \delta)$.

- The [R] matrix can be determined using Wheeler's incremental inductance rule [21] or perturbation theory [22].

Although Eqs. (4) and (5) provide unique definitions of the four circuit parameters, it is possible to cast them into another form which is more convenient for many purposes, as is done by Brews [23]. A simpler derivation shows that the circuit parameters are given exactly by

$$
\begin{gathered}
C=\frac{1}{\left|Z_{0} I\right|^{2}}\left[\int_{S} \varepsilon^{r}\left|E_{t}\right|^{2} d S-\int_{S} \mu^{r}\left|H_{l}\right|^{2} d S\right] \\
L=\frac{1}{|I|^{2}}\left[\int_{S} \mu^{r}\left|H_{t}\right|^{2} d S-\int_{S} \varepsilon^{r}\left|E_{l}\right|^{2} d S\right] \\
G=\frac{\omega}{\left|Z_{0} I\right|^{2}}\left[\int_{S} \varepsilon^{i}\left|E_{t}\right|^{2} d S+\int_{S} \mu^{i}\left|H_{l}\right|^{2} d S\right] \\
R=\frac{\omega}{|I|^{2}}\left[\int_{S} \mu^{i}\left|H_{t}\right|^{2} d S+\int_{S} \varepsilon^{i}\left|E_{l}\right|^{2} d S\right]
\end{gathered}
$$


where $E_{t}, H_{t}$ and $E_{1}, H_{1}$ are the transverse and longitudinal components of electric and magnetic fields, respectively. Here $\varepsilon \equiv \varepsilon^{r}-j \varepsilon^{i}$ and $\mu \equiv \mu^{r}-j \mu^{i}$. In passive media, the four real components $\varepsilon^{\mathrm{r}}, \varepsilon^{\mathrm{i}}, \mu^{\mathrm{r}}$, and $\mu^{\mathrm{i}}$ are all nonnegative. Metal conductivity is not included as an explicit term in $\varepsilon$ but is instead absorbed in $\varepsilon^{i}$. In general, of course, $\varepsilon$ and $\mu$ depend on $\omega$.

Equations (9) through (12) have many applications. In addition to providing a means of numerically calculating the circuit parameters from known fields, they offer opportunities for analytical calculations and approximations as well. The quadratic form in which the fields appear make them particularly useful for these purposes. Another major role they serve is in the attribution of circuit-parameter components to portions of the cross-section. For example, it is common to divide the inductance $\mathrm{L}$ into an "external" inductance in the dielectric and an "internal" inductance in the imperfect metal. Such a division cannot be undertaken using only Eqs. (4) and (5) but is readily obtainable by dividing the surface integral in Eq. (10) into dielectric and metal regimes.

An interconnect system tends to require complex modelling because the conductor material is not lossless and the lines can be coupled both capacitively and inductively. The finite conductivity of the conductor results in a variation of the current density distribution in the conductor. Skin effect loss (conductor loss) and dielectric losses as well as different signal velocities at high frequencies make the interconnect resistance and capacitance frequency dependent.

\section{CALCULATION OF THE CAPACITANCE MATRIX}

For our purposes, the geometry may be restricted to $\mathrm{N}_{\mathrm{c}}$ interconnect lines with zero or finite thickness embedded in $\mathrm{L}$ dielectric layers, as shown in Figure 3a. The use of a complex permittivity allows to take dielectric layers as well as high-loss conductor layers into account. Element $\mathbf{P}_{\mathrm{ij}}$ of the coefficient-of-potential matrix may be calculated as the potential $\varphi_{\mathrm{i}}$ on the conductor (i) when the charge on the conductor

(j) is 1 and $0 \mathrm{C}$ on all other conductors. The total charge on conductor (j) is the integral of the surface charge distribution $\rho_{j}\left(r_{s}\right)$ over the surface of the conductor $(j): Q_{j}=\oint_{j} \rho_{j}\left(r_{s}\right) d S_{j}$. The potential distribution 
in any point $\mathbf{r}_{\mathrm{p}}$ due to the charge distribution $\rho_{\mathrm{j}}\left(\mathbf{r}_{\mathrm{s}}\right)$ on all conductors $\left(\mathrm{j}=1, \ldots, \mathrm{N}_{\mathrm{c}}\right)$, can be expressed as

$$
\varphi\left(\mathbf{r}_{p}\right)=\sum_{j=1}^{N_{c}} \oint_{j} G\left(\mathbf{r}_{p} ; \mathbf{r}_{s}\right) \rho_{j}\left(\mathbf{r}_{s}\right) d S_{j} .
$$

In this integral, $G\left(r_{p} ; r_{s}\right)$ is the space-domain Green's function of the layered medium (see Section 4). It gives the voltage at the position $\mathbf{r}_{\mathbf{p}}$ due to a Dirac point charge at the position $\mathbf{r}_{\mathbf{s}}$. While Eq. (13) establishes a global relationship between the potential and charge for a multiconductor system, there exists a similar relationship among the interconnect conductors. This is written in matrix notation as:

$$
[\mathrm{V}]=[\mathrm{G}][\mathrm{Q}]
$$

where $[\mathrm{V}]=\left[\mathrm{V}_{1}, \mathrm{~V}_{2}, \ldots, \mathrm{V}_{\mathrm{Nc}}\right]^{\mathrm{T}}$ and $[\mathrm{Q}]=\left[\mathrm{Q}_{1}, \mathrm{Q}_{2}, \ldots, \mathrm{Q}_{\mathrm{Nc}}\right]^{\mathrm{T}}$ collect the conductor voltages and total charges, respectively, and [G] is an $N_{c} \times N_{c}$ matrix whose entries $G_{i j}$ are called coefficients-of-potential. It can be shown later that $[\mathrm{G}]$ is a symmetric matrix.

The inverse $[\mathrm{C}]$ of $[\mathrm{G}]$ is the so-called capacitance matrix of the system of conductors in the structure. It expresses the charge $Q_{i}$ on conductor $i$ as a function of the voltages $\mathrm{V}_{1}, \ldots, \mathrm{V}_{\mathrm{Nc}}$ :

$$
[\mathrm{Q}]=[\mathrm{C}][\mathrm{V}] \text {. }
$$

The entries $C_{i j}$ of $[C]$ is the charge on the conductor $i$ when the $j$-th conductor is held at unit potential and all other conductors are shortcircuited to ground.

It is clear that we must begin by finding the space-domain Green's function of the layered dielectric medium.

\section{DERIVATION OF THE MULTILAYER GREEN'S FUNCTION}

Here, we consider the problem of finding multilayer dielectric Green's functions for the interconnects with rectangular boundary surfaces (planar layered structures).

The Green function of the medium $\mathrm{G}\left(\mathbf{r}_{p} ; \mathbf{r}_{s}\right)$ (see Fig. 3b) is the potential at any point $\mathbf{r}_{p}$ due to a Dirac charge placed at point $\mathbf{r}_{s}$ in the 
top layer and is given as a solution of the Poisson equation:

$$
\nabla^{2} G\left(x_{p}, y_{p}, z_{p} ; x_{s}, y_{s}, z_{s}\right)=-\frac{\delta\left(x_{p}-x_{s}\right) \delta\left(y_{p}-y_{s}\right) \delta\left(z_{p}-z_{s}\right)}{\varepsilon_{L}} .
$$

Let $G=X\left(x_{p}, x_{s}\right) Y\left(y_{p}, y_{s}\right) Z_{1}\left(z_{p}, z_{s}\right)$. Substituting in the above equation, we have

$$
Y Z_{1} \frac{d^{2} X}{d x_{p}^{2}}+X Z_{1} \frac{d^{2} Y}{d y_{p}^{2}}+X Y \frac{d^{2} Z_{1}}{d z_{p}^{2}}=-\frac{\delta\left(x_{p}-x_{s}\right) \delta\left(y_{p}-y_{s}\right) \delta\left(z_{p}-z_{s}\right)}{\varepsilon_{L}} .
$$

\section{Assume}

$$
X\left(x_{p}, x_{s}\right)=\cos \left(m_{1} \pi x_{p} / a\right) ; \quad Y\left(y_{p}, y_{s}\right)=\cos \left(n_{1} \pi y_{p} / b\right)
$$

where $\mathrm{m}_{1} \in[0, \propto), n_{1} \in[0, \propto)$, to satisfy the boundary conditions of zero normal electric field on the side walls. We get from (17)

$$
\begin{gathered}
\sum_{m_{1}=0}^{\infty} \sum_{n_{1}=0}^{\infty} \cos \frac{m_{1} \pi x_{p}}{a} \cos \frac{n_{1} \pi y_{p}}{b}\left\{\frac{d^{2} Z_{1}}{d z_{p}^{2}}-\left[\left(\frac{m_{1} \pi}{a}\right)^{2}+\left(\frac{n_{1} \pi}{b}\right)^{2}\right] Z_{1}\right\} \\
=-\frac{\delta\left(x_{p}-x_{s}\right) \delta\left(y_{p}-y_{s}\right) \delta\left(z_{p}-z_{s}\right)}{\varepsilon_{L}}
\end{gathered}
$$

Multiplying both sides by $\cos \left(m \pi x_{p} / a\right) \cos \left(n \pi y_{p} / b\right)$ and integrating over $x_{p}$ and $y_{p}$ from 0 to $a$ and 0 to $b$, respectively, we get

$$
\begin{aligned}
\frac{a b}{4}\{ & \left.\frac{d^{2} Z_{1}}{d z_{p}^{2}}-\left[\left(\frac{m \pi}{a}\right)^{2}+\left(\frac{n \pi}{b}\right)^{2}\right] Z_{1}\right\} \\
& =-\frac{\delta\left(z_{p}-z_{s}\right)}{\varepsilon_{L}} \cos \left(\frac{m \pi x_{s}}{a}\right) \cos \left(\frac{n \pi y_{s}}{b}\right) .
\end{aligned}
$$

Let $Z_{1}=Z\left(z_{p}, z_{s}\right) \cos \left(m \pi x_{s} / a\right) \cos \left(n \pi y_{s} / b\right)$. If we substitute in relation (18), we get

$$
\frac{a b}{4}\left[\frac{d^{2}}{d z_{p}^{2}}-t_{m n}^{2}\right] Z\left(z_{p}, z_{s}\right)=-\frac{\delta\left(z_{p}-z_{s}\right)}{\varepsilon_{L}}
$$


where $t_{m n}^{2}=(m \pi / a)^{2}+(n \pi / b)^{2}$. For $z_{p} \neq z_{s}$ the right hand side of (19) is zero. A general solution to (19) would be of the form

$$
Z\left(z_{p}, z_{s}\right)=C_{p} \exp \left(t_{m n} z_{p}\right)+D_{p} \exp \left(-t_{m n} z_{p}\right) .
$$

We assume that the source point and the field point are in the top layer that is $d_{L-1} \leq z_{p}, z_{s} \leq d_{L}$. Now we consider the solution for $Z$ in the region $z_{p} \leq z_{s}$ (in any layer in the structure). Since the ground plane has been assumed to be at zero potential, we have $\left.Z\left(z_{p}, z_{s}\right)\right|_{z_{p}=0}=0$. At the interface between dielectric layers $\varepsilon_{\mathrm{k}}$ and $\varepsilon_{\mathrm{k}+1}$, two boundary conditions need to be satisfied, i.e., the potential and the normal component of electric induction vector must be continuous. Specifically, the set of equations relating $\left(C_{k+1}, D_{k+1}\right)$ to $\left(C_{k}, D_{k}\right)$ can bc represented in the matrix form (are computed recursively)

$$
\left[\begin{array}{c}
C_{k+1} \\
D_{k+1}
\end{array}\right]=[A]\left[\begin{array}{l}
C_{k} \\
D_{k}
\end{array}\right]
$$

where matrix $[A]$ has the form

$$
[A]=\left[\begin{array}{cc}
\frac{1}{2}\left(1+\frac{\varepsilon_{k}}{\varepsilon_{k+1}}\right) & \frac{1}{2}\left(1-\frac{\varepsilon_{k}}{\varepsilon_{k+1}}\right) \exp \left(-2 t_{m n} d_{k}\right) \\
\frac{1}{2}\left(1-\frac{\varepsilon_{k}}{\varepsilon_{k+1}}\right) \exp \left(2 t_{m n} d_{k}\right) & \frac{1}{2}\left(1+\frac{\varepsilon_{k}}{\varepsilon_{k+1}}\right)
\end{array}\right]
$$

where $1 \leq k \leq L$ and $\mathrm{C}_{1}=1, \mathrm{D}_{1}=-1$.

In the top layer (source layer), we have

$$
Z_{l}=C_{s} \exp \left(t_{m n} z_{p}\right)+D_{s} \exp \left(-t_{m n} z_{p}\right)
$$

where $d_{L-1} \leq z_{p} \leq z_{s} \leq d_{L}$. The field must satisfy the zero normal electric induction condition at $z_{p}=d_{L}$. Thus in the region $z_{s} \leq z_{p} \leq d_{L}$, the potential must be of the form

$$
Z_{u}=\exp \left(t_{m n} z_{p}\right)+\exp \left(-t_{m n}\left(z_{p}-2 d_{L}\right) .\right.
$$

Applying symmetry on the function $\mathrm{Z}\left(\mathrm{z}_{\mathrm{p}}, \mathrm{z}_{\mathrm{s}}\right)$, i.e., requiring that $\mathrm{Z}$ must be symmetric under the interchange of source and field points, we see that $Z_{u}$ and $Z_{1}$ must be of the form

$$
\begin{aligned}
Z_{u}=K & \left\{C_{s} \exp \left(t_{m n} z_{s}\right)+D_{s} \exp \left(-t_{m n} z_{s}\right)\right\} \\
& \left\{\exp \left(t_{m n} z_{p}\right)+\exp \left(-t_{m n}\left(z_{p}-2 d_{L}\right)\right)\right\}
\end{aligned}
$$




$$
\begin{array}{r}
Z_{l}=K\left\{C_{s} \exp \left(t_{m n} z_{p}\right)+D_{s} \exp \left(-t_{m n} z_{p}\right)\right\} \\
\left\{\exp \left(t_{m n} z_{s}\right)+\exp \left(-t_{m n}\left(z_{s}-2 d_{L}\right)\right)\right\}
\end{array}
$$

We can observe that Eqs. (25a) and (25b) satisfy the requirements that the potential must be continuous at $z_{p}=z_{s}$, and to determine the constant $K$, relation (19) is integrated over discontinuity at $z_{p}=z_{s}$, which yields (letting $\delta \rightarrow 0$, subsequently)

$$
\left.\frac{d Z}{d z_{p}}\right|_{z_{p}=z_{s}+\delta}-\left.\frac{d Z}{d z_{p}}\right|_{z_{p}=z_{s}-\delta}=-\frac{4}{a b \varepsilon_{s}} .
$$

At $\mathrm{z}_{\mathrm{p}}=\mathrm{z}_{\mathrm{s}}+\delta, \mathrm{Z}$ is given by $\mathrm{Z}_{\mathrm{u}}$ while at $\mathrm{z}_{\mathrm{p}}=\mathrm{z}_{\mathrm{s}}-\delta$ is given by $\mathrm{Z}_{\mathrm{l}}$. Substituting (25a) and (25b) in (26) and solving for $K$, we get

$$
K=\frac{2}{a b \varepsilon_{s} t_{m n}\left(C_{s} \exp \left(2 t_{m n} d_{L}\right)-D_{s}\right)}
$$

Thus for $m>0$ and $n>0$, we have

$$
\begin{aligned}
(G)_{m, n} & >0 \\
= & \sum_{m=1}^{\infty} \sum_{n=1}^{\infty} 2 f_{m n} \cos \left(\frac{m \pi x_{p}}{a}\right) \cos \left(\frac{m \pi x_{s}}{a}\right) \cos \left(\frac{n \pi y_{p}}{b}\right) \cos \left(\frac{n \pi y_{s}}{b}\right) .
\end{aligned}
$$

with

$f_{m_{n}}=\frac{\left(C_{p} \exp \left(t_{m_{n}} z_{p}\right)+D_{p} \exp \left(-t_{m_{n}} z_{p}\right)\right)\left(\exp \left(t_{m_{n}}\left(d_{L}-z_{s}\right)\right)+\exp \left(-t_{m_{n}}\left(d_{L}-z_{s}\right)\right)\right)}{a b t_{m_{n}} \varepsilon_{s}\left(C_{s} \exp \left(t_{m_{n}} d_{L}\right)-D_{s} \exp \left(-t_{m_{n}} d_{L}\right)\right)}$.

For the case when $\mathrm{m}=0, \mathrm{n}>0$ or $\mathrm{m}>0, \mathrm{n}=0$, the form (28) remains the same except that the multiplicative constant 2 is replaced by 1 . For the case $m=n=0$ we see from Eq. (19) that the general solution for the function $Z$ must be of the form $Z=C_{p} Z_{p}+D_{p}$. Following a similar argument as presented in the derivation of (28), $Z_{1}$ in the bottom dielectric layer with $\varepsilon_{1}$ is given by (applying the boundary condition on ground plane we see that $D_{1}=0$ )

$$
Z_{l}=w\left(z_{s}\right) z_{p} .
$$


The function $Z_{1}$ in any layer $p$, will be given by

$$
Z_{l}=w\left(z_{s}\right)\left(C_{p} z_{p}+D_{p}\right) .
$$

Equating the potential and the normal component of electric induction vector at every interface, we have the following matrix relation between $\left(C_{k+1}, D_{k+1}\right)$ and $\left(C_{k}, D_{k}\right)$

$$
\left[\begin{array}{l}
C_{k+1} \\
D_{k+1}
\end{array}\right]=\left[\begin{array}{cc}
\frac{\varepsilon_{k}}{\varepsilon_{k+1}} & 0 \\
\left(1-\frac{\varepsilon_{k}}{\varepsilon_{k+1}}\right) d_{k} & 1
\end{array}\right]\left[\begin{array}{l}
C_{k} \\
D_{k}
\end{array}\right]
$$

where $C_{1}=1, D_{1}=0$.

The solution for $\mathrm{Z}_{\mathrm{u}}$ (where $\mathrm{z}_{\mathrm{s}} \leq \mathrm{z}_{\mathrm{p}}$ ), must be of the form $Z_{u}=q\left(z_{s}\right)$. Applying the symmetry argument, we get (in the source layer)

$$
\begin{aligned}
& Z_{l}=K\left(C_{s} z_{p}+D_{s}\right) \\
& Z_{u}=K\left(C_{s} z_{s}+D_{s}\right)
\end{aligned}
$$

Integrating Eq. (19) over the discontinuity at $z_{p}=z_{s}$, we get

$$
K=\frac{1}{a b \varepsilon_{s} C_{s}} .
$$

Thus, for $\mathrm{m}=\mathrm{n}=0$, we get

$$
(G)_{m=n=0}=\frac{1}{a b \varepsilon_{s} C_{s}}\left(C_{p} z_{p}+D_{p}\right) .
$$

Using the Eqs. (28) and (35), the complete expression for Green's function of the problem considered here, is

$$
\begin{gathered}
G=(G)_{m=n=0}+\sum_{m=0}^{\infty} \sum_{n=0}^{\infty} H_{m n} f_{m n} \cos \left(\frac{m \pi x_{p}}{a}\right) \cos \left(\frac{m \pi x_{s}}{a}\right) \\
\cos \left(\frac{n \pi y_{p}}{b}\right) \cos \left(\frac{n \pi y_{s}}{b}\right)
\end{gathered}
$$

where

$$
H_{m n}= \begin{cases}0, & m=n=0 \\ 1, & m>0, n=0 \text { or } m=0, n>0 \\ 2, & m>0, n>0\end{cases}
$$


The Green's function derived above is represented as a double infinite series which converges quickly. In practical applications, a good accuracy of the results is achieved using the low number of the modes in the new multilayer Green's function.

\section{SOLUTION OF THE BOUNDARY INTEGRAL EQUATION}

A formal solution of the Poisson equation was given by Eq. (13). Since the charge density is only present on the surface of the interconnect conductors, according to the equivalent source principle for electromagnetic field, we can replace the strip conductors with a piece of surface charge density distribution $\rho_{\mathrm{s}}\left(\mathbf{r}_{\mathrm{s}}\right)$ around the surface $\mathrm{S}$, as shown in Figure 4. Using a Green's function (36), we can get a boundary integral equation on the surface as follows:

$$
\varphi\left(\mathbf{r}_{p}\right)=\oint_{S} \rho_{s}\left(\mathbf{r}_{s}\right) G\left(\mathbf{r}_{p} ; \mathbf{r}_{s}\right) d S_{s}
$$

The computational tool that we shall use belongs to the class of finite element procedures. It is specifically known as method of moments [17]. With this method, the domain of the function $f$ to be

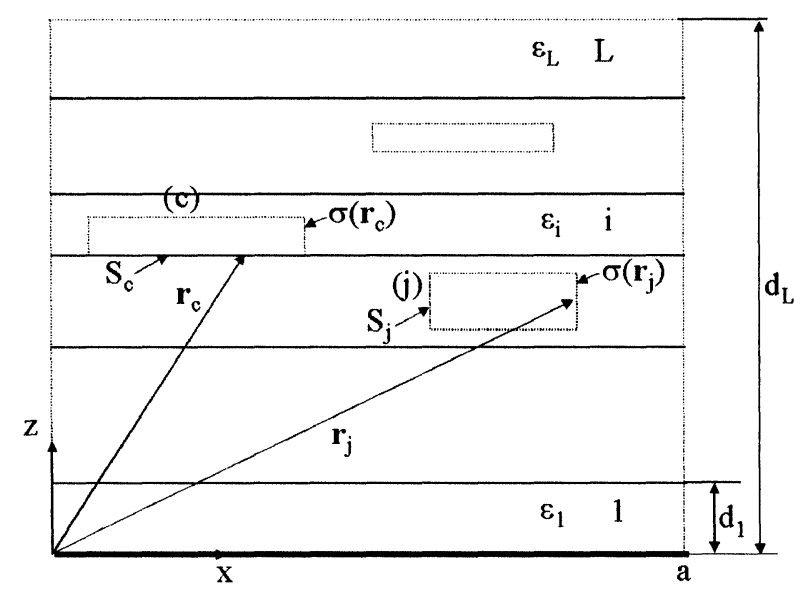

FIGURE 4 Charge density distribution on the discretized surface of the conductors. 
approximated is partitioned into a number of sub-domains. A local approximation of $f$ is defined on each sub-domain, such that their assembly is a piecewise approximation of $\mathrm{f}$.

In our case, the function of surface charge density $\rho_{\mathrm{s}}\left(\mathbf{r}_{\mathrm{s}}\right)$ is approximated as

$$
\rho_{s}\left(\mathbf{r}_{s}\right) \cong \sum_{i=1}^{N_{s}} \rho_{s i} f_{i}\left(\mathbf{r}_{s}\right) .
$$

Hence, the $f_{i}$ 's are $N_{s}$ independent shape functions known as basis or expansion functions, which are non-zero only on a part $S_{i}$ of $S$ $\left(i=1, \ldots, N_{s}\right)$ and which have unity weight. In particular:

$$
\int_{S_{i}} f_{i}\left(\mathbf{r}_{s}\right) d S= \begin{cases}1 & \text { if } i=j \\ 0 & \text { if } i \neq j\end{cases}
$$

We then obtain an approximation $\varphi^{\mathrm{a}}\left(\mathbf{r}_{\mathbf{p}}\right)$ of $\varphi\left(\mathbf{r}_{\mathbf{p}}\right)$ as follows:

$$
\begin{aligned}
\varphi^{a}\left(\mathbf{r}_{p}\right) & =\int_{S} G\left(\mathbf{r}_{\mathbf{p}} ; \mathbf{r}_{\mathbf{s}}\right) \sum_{i=1}^{N_{s}} \rho_{s i} f_{i}\left(\mathbf{r}_{s}\right) d S_{s} \\
& =\sum_{i=1}^{N_{s}} \rho_{s i} \int_{S_{i}} G\left(\mathbf{r}_{p} ; \mathbf{r}_{s}\right) f_{i}\left(\mathbf{r}_{s}\right) d S_{s}
\end{aligned}
$$

The $\rho_{\mathrm{si}}$ 's are then $\mathrm{N}_{\mathrm{s}}$ independent constants to be determined so as to obtain a good approximation of $\varphi\left(\mathbf{r}_{\mathrm{p}}\right)$. For $\mathrm{N}_{\mathrm{s}}$ unknown constants, we need $\mathrm{N}_{\mathrm{s}}$ independent equations. These can be obtained by using the theory of the method of moments [17]. For that purpose, Eq. (41) can be written as

$$
\varphi^{a}\left(\mathbf{r}_{p}\right)=\sum_{i=1}^{N_{s}} \rho_{s i} W_{i}\left(\mathbf{r}_{p}\right)
$$

where $\mathrm{W}_{\mathrm{i}}\left(\mathbf{r}_{\mathrm{p}}\right)=\int_{\mathrm{Si}} \mathrm{G}\left(\mathbf{r}_{\mathrm{p}} ; \mathbf{r}_{\mathrm{s}}\right) \mathrm{f}_{\mathrm{i}}\left(\mathbf{r}_{\mathrm{s}}\right) \mathrm{d} \mathrm{S}_{\mathrm{s}}$. The general method of moments formulation of the Eq. (42) would be written as:

$$
\begin{aligned}
\left\langle\varphi\left(\mathbf{r}_{p}\right), w_{j}\left(\mathbf{r}_{p}\right)\right\rangle & =\left\langle\varphi^{a}\left(\mathbf{r}_{p}\right), w_{j}\left(\mathbf{r}_{p}\right)\right\rangle \\
& =\left\langle\sum_{i=1}^{N_{s}} \rho_{s i} W_{i}\left(\mathbf{r}_{p}\right), w_{j}\left(\mathbf{r}_{p}\right)\right\rangle \\
& =\sum_{i=1}^{N_{s}} \rho_{s i}\left\langle W_{i}\left(\mathbf{r}_{p}\right), w_{j}\left(\mathbf{r}_{p}\right)\right\rangle, \quad \mathrm{j}=1, \ldots, \mathbf{N}_{s}
\end{aligned}
$$


where $\langle$,$\rangle is a suitable bi-linear product and w_{j}\left(\mathbf{r}_{p}\right), j=1, \ldots, \mathrm{N}_{\mathrm{s}}$, is a set of weighting functions aimed at "averaging out" the approximation error. The weighting functions are defined on the domain of $\varphi\left(\mathbf{r}_{\mathbf{p}}\right)$, that is the surface of the conductors. The solution of the simultaneous equations results in the desired linear combination of the $\mathrm{N}_{\mathbf{s}}$ independent basis functions $\mathbf{f}_{\mathbf{i}}$.

A suitable bi-linear product for our problem is [17]

$$
\left\langle\varphi\left(\mathbf{r}_{p}\right), w_{j}\left(\mathbf{r}_{p}\right)\right\rangle=\int_{S} \varphi\left(\mathbf{r}_{p}\right) w_{j}\left(\mathbf{r}_{p}\right) d S_{p}
$$

where the integral extends over the conductor surfaces.

Suitable weighting functions are defined below, but in the case of boundary integral method they are also defined in a piecewise fashion, like the expansion or basis functions. In that case, $w_{j}\left(\mathbf{r}_{p}\right)$ is non-zero only on the part $S_{j}$ of $S$. Hence, Eq. (43) can be written in matrix notation as

$$
[\Phi]=[G]\left[\rho_{s}\right]
$$

where $[\Phi]=\left[\Phi_{1}, \Phi_{2}, \ldots, \Phi_{\mathrm{Ns}}\right]^{\mathrm{T}},\left[\rho_{\mathrm{s}}\right]=\left[\rho_{\mathrm{s} 1}, \rho_{\mathrm{s} 2}, \ldots, \rho_{\mathrm{sNs}}\right]^{\mathrm{T}}$ and

$$
\begin{gathered}
\Phi_{j}=\left\langle\varphi\left(\mathbf{r}_{p}\right), w_{j}\left(\mathbf{r}_{p}\right)\right\rangle=\int_{S_{j}} \varphi\left(\mathbf{r}_{p}\right) w_{j}\left(\mathbf{r}_{p}\right) d S_{p} \\
G_{j i}=\left\langle W_{i}\left(\mathbf{r}_{p}\right), w_{j}\left(\mathbf{r}_{p}\right)\right\rangle=\int_{S_{j}} \int_{S_{i}} G\left(\mathbf{r}_{p} ; \mathbf{r}_{s}\right) w_{j}\left(\mathbf{r}_{p}\right) f_{i}\left(\mathbf{r}_{s}\right) d S_{s} d S_{p} .
\end{gathered}
$$

A convenient choice for the weighting functions is $w_{j}=f_{i}$, i.e., the weighting functions are made equal to the expansion or basis functions. This is known as Galerkin procedure. A Galerkin solution leads to symmetric matrices, which is attractive from the computational point of view as well as from a physical one, since the physical system is symmetrical.

With Galerkin's procedure, Eq. (45) reduces to

$$
\Phi_{j}=\varphi\left(\mathbf{r}_{j}\right)
$$

where $\mathbf{r}_{\mathbf{j}}$ is some point on $\mathrm{S}_{\mathbf{j}}$, because the potential is constant over the each discrete element and because $\int_{S_{j}} w_{j}\left(\mathbf{r}_{p}\right) d S_{s}=1$ since the weighted functions are normalized. Equation (46) reduces in the case of 
Galerkin's procedure to

$$
G_{j i}=G_{i j}=\int_{S_{j}} \int_{S_{i}} G\left(\mathbf{r}_{p} ; \mathbf{r}_{s}\right) f_{j}\left(\mathbf{r}_{p}\right) f_{i}\left(\mathbf{r}_{s}\right) d S_{s} d S_{p}
$$

After having established a calculation procedure for solving Eq. (13), the remaining problem is to relate the solution to the multilayer IC interconnect capacitances. In order to do that, we define an incidence matrix [ $I_{M}$ ] with $N_{t s}$ rows ( $N_{t s}$ is the total number of the discretized elements in the system) and $N_{c}$ columns $\left(N_{c}\right.$ is the number of conductors). Since, in general, each conductor contains many discretized elements, $\left[I_{M}\right]$ is non-square with $N_{t s} \gg N_{c}$. [ $\left.I_{M}\right]$ is a given value as follows:

$$
I_{M i j}= \begin{cases}1, & \text { if discretized element } i \text { is on conductor } j \\ 0, & \text { otherwise }\end{cases}
$$

First, bear in mind that $[\mathrm{Q}]$ is the vector of the conductor charges. The charge on a conductor is the sum of all elemental charges, which can be expressed as

$$
[Q]=\left[I_{M}\right]^{T}\left[\rho_{s}\right]
$$

Second, bear in mind that [V] is the vector of the conductor potentials. In quasi-static case, the element voltages are the same for all elements on a conductor (surface of the conductor is equipotential). Hence, [V] and $[\Phi]$ are related as follows:

$$
[\Phi]=\left[I_{M}\right][V]
$$

Then using Eqs. (44), (50) and (51), we have

$$
[Q]=\left[I_{M}\right]^{T}\left[\rho_{s}\right]=\left[I_{M}\right]^{T}[G]^{-1}[\Phi]=\left[I_{M}\right]^{T}[G]^{-1}\left[I_{M}\right][V] .
$$

By comparing this with Eq. (15), we find

$$
[C]=\left[I_{M}\right]^{T}[G]^{-1}\left[I_{M}\right]
$$

which is the desired result. 


\section{DISCUSSION OF THE RESULTS}

In our approach suggested in this paper, regions of different complex permittivities are modeled by using the multilayer Green function (charge point) to incorporate the effects of ground planes (electric or magnetic type) and dielectric interfaces. The advantages of using such an approach are that only conductor surfaces need to be discretized and that there is no need to truncate wide ground conductors and dielectric layers. Therefore the system matrix is reduced in size. The Green's function may be easily found for planar layered interconnect configurations and other VLSI and MCMs structures in which all dielectric interfaces and ground planes are planar, parallel and infinite in extent.

A computer program based on the expressions presented in this paper, has been developed to analyze the different multilayer interconnect configurations with strip conductors of zero or finite thickness.

This section has the main goal to make a comparison between the results of our model and one of other models available in the literature.

Example 1 The aim of this example is to illustrate the accuracy of the approach presented, by making a comprehensive comparisons with respect to the per unit length capacitance of an open coplanar interconnect configuration. As shown in Figure 5, the electrical and geometrical parameters of this configuration are:

- four line interconnect system: $\varepsilon_{\mathrm{r}}=9.8, \mathrm{w}_{1}=\mathrm{w}_{2}=\mathrm{w}_{3}=\mathrm{w}_{4}=\mathrm{w}$, $d_{12}=d_{23}=d_{34}=d, w / h=0.102, d / h=0.173$;

- five-line interconnect system: $\varepsilon_{\mathrm{r}}=2.5, \mathrm{~h}=800 \mu \mathrm{m}, \mathrm{w}_{1}=\mathrm{w}_{3}=\mathrm{w}_{5}=$ $160 \mu \mathrm{m}, \mathrm{w}_{2}=\mathrm{w}_{4}=470 \mu \mathrm{m}, \mathrm{d}_{12}=\mathrm{d}_{23}=\mathrm{d}_{34}=\mathrm{d}_{45}=385 \mu \mathrm{m}$.

Based on the uniform segmentation principle, numerical results are produced for a number of basis functions to model the charge density distribution on each conductor $\mathrm{N}_{\mathrm{b}}=11$. This integer is chosen by increasing the number of subsections until the resulting capacitance does not vary by more than $0.01 \%$. In Tables Ia, $b$, results obtained using the approach described above, are compared with those obtained in [24] by using the simplified parameter dependence of the inverted capacitance coefficient matrix and with those obtained in [25] using the method of lines, respectively. Upon examining the three sets 


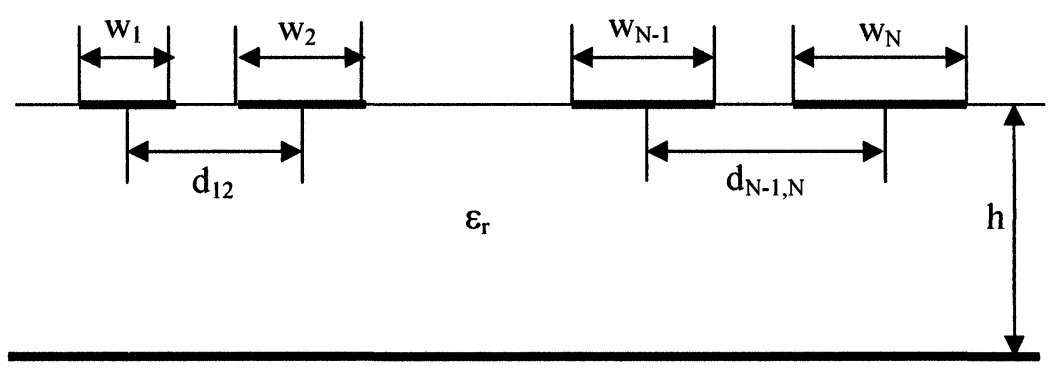

FIGURE 5 Geometry of the structure for Example 1 with four- and five-line system.

TABLE Ia Capacitance matrix of four-line system

\begin{tabular}{lccc}
\hline Capacitance & MoL [25] & Paper [24] & This approach \\
$C_{i j}$ & 4.7965 & 4.963 & 4.7538 \\
$\mathrm{C}_{10}$ & 5.8084 & 5.851 & 5.8231 \\
$\mathrm{C}_{12}$ & 1.1476 & 0.961 & 1.1432 \\
$\mathrm{C}_{13}$ & 0.6184 & 0.468 & 0.6180 \\
$\mathrm{C}_{14}$ & 2.8953 & 2.781 & 2.8876 \\
$\mathrm{C}_{20}$ & 5.3306 & 5.429 & 5.3370 \\
$\mathrm{C}_{23}$ & & & \\
\hline
\end{tabular}

TABLE Ib Capacitance matrix of five-line system

\begin{tabular}{lccc}
\hline $\begin{array}{l}\text { Capacitance } \\
C_{i j}\end{array}$ & MoL [25] & Paper [24] & This approach \\
\hline $\mathrm{C}_{10}$ & 1.8009 & 1.878 & 1.7989 \\
$\mathrm{C}_{12}$ & 2.5889 & 2.250 & 2.5982 \\
$\mathrm{C}_{13}$ & 0.0880 & 0.0376 & 0.0876 \\
$\mathrm{C}_{14}$ & 0.0791 & 0.0917 & 0.0789 \\
$\mathrm{C}_{15}$ & 0.0229 & 0.0361 & 0.0228 \\
$\mathrm{C}_{20}$ & 2.2502 & 2.354 & 2.2511 \\
$\mathrm{C}_{23}$ & 2.3511 & 2.062 & 2.3521 \\
$\mathrm{C}_{24}$ & 0.5720 & 0.553 & 0.5745 \\
$\mathrm{C}_{30}$ & 0.8524 & 0.851 & 0.8550 \\
\hline
\end{tabular}

of data, it may be concluded that the inaccuracy of the solution presented here is less than $1 \%$ (compared with the method of lines) for most of applicable range of physical dimensions used in IC and MCMs and available dielectric materials $\left(1<\varepsilon_{\mathrm{r}}<15\right)$.

Example 2 Consider a triple coupled lines structure shown in Figure 6. This configuration of coplanar waveguides is used for the 


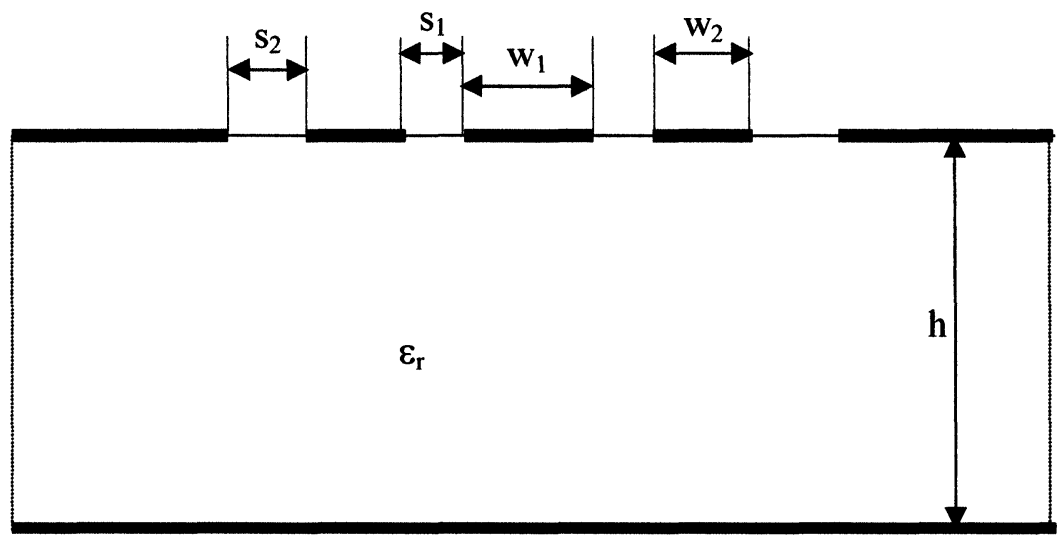

FIGURE 6 Triple coupled CPW lines.

design of hybrid, IC and monolithic integrated circuits, therefore it becomes highly desirable to have an efficient and reliable method in obtaining the per unit length transmission line parameters. To analyze this structure, all conductors are assumed to be infinitely thin and perfectly conducting. The number of the subsections used on the middle conductor is 7 and the number on two symmetric side conductors is 5 . Numerical values of the capacitances $\mathrm{C}_{11}, \mathrm{C}_{12}, \mathrm{C}_{22}$ and $\mathrm{C}_{13}$, generated by the proposed approach and by a CAD-oriented analytical solution [26], are given in Table II. The technological and geometrical parameters of this structure are: $\varepsilon_{\mathrm{r}}=12.9, \mathrm{w}_{1} / \mathrm{h}=0.2$ and $\mathrm{s}_{1} / \mathrm{w}_{1}=1$. Note that the discrepancies between the calculated values by the two different procedures are less than $1 \%$, over a wide range of technological and geometrical parameters.

TABLE II Triple coupled CPW lines with lower ground plane

\begin{tabular}{|c|c|c|c|c|c|c|c|c|c|}
\hline \multirow[b]{2}{*}{$s_{2} / w_{1}$} & \multirow[b]{2}{*}{$w_{2} / w_{1}$} & \multicolumn{4}{|c|}{$\begin{array}{c}\text { This approach } \\
\text { capacitance }[p F]\end{array}$} & \multicolumn{4}{|c|}{$\begin{array}{c}\text { Analytical solution }[26] \\
\text { capacitance }[p F]\end{array}$} \\
\hline & & $C_{11}$ & $C_{22}$ & $C_{12}$ & $C_{13}$ & $C_{11}$ & $C_{22}$ & $C_{12}$ & $C_{13}$ \\
\hline 0.5 & 0.5 & 158.3 & 194.2 & 58.29 & 5.148 & 158.4 & 194.1 & 58.25 & 5.153 \\
\hline 0.5 & 2 & 241.2 & 194.9 & 74.40 & 13.59 & 241.1 & 194.9 & 74.45 & 13.60 \\
\hline 1 & 0.5 & 145.4 & 192.9 & 60.80 & 6.25 & 145.4 & 193.0 & 60.84 & 6.20 \\
\hline 1 & 2 & 221.7 & 194.5 & 75.05 & 14.31 & 221.8 & 194.8 & 75.16 & 14.31 \\
\hline 2 & 0.5 & 137.0 & 191.2 & 63.15 & 7.269 & 137.1 & 191.5 & 63.25 & 7.274 \\
\hline 2 & 2 & 207.6 & 194.5 & 75.85 & 15.19 & 207.5 & 194.7 & 75.99 & 15.21 \\
\hline
\end{tabular}


The proposed approach is both easy to implement and reliable (is semianalytical procedure), thus making it an excellent choice for use in CAD-oriented design tools.

Example 3 Let us consider a pair of coupled parallel interconnection lines embedded in a two-layered dielectric region with structure as shown in Figure 7. 32 segments are used for the conductor-to-dielectric interfaces to calculate the capacitance matrix (as in the paper [28]). Table III provides a comparison of the capacitances per unit length between the data obtained by our technique and the data obtained by two different methods in the papers [22,28], respectively. Good agreement between our results and the published data can be seen from this table. Note that the discrepancies between the values calculated by our approach and the one of total charge Green's function method [22] are practically smaller than $0.2 \%\left(\mathrm{C}_{12}\right)$, while the Pade approximation of [28] differs up to $4 \%\left(\mathrm{C}_{12}\right)$. It is clear that the

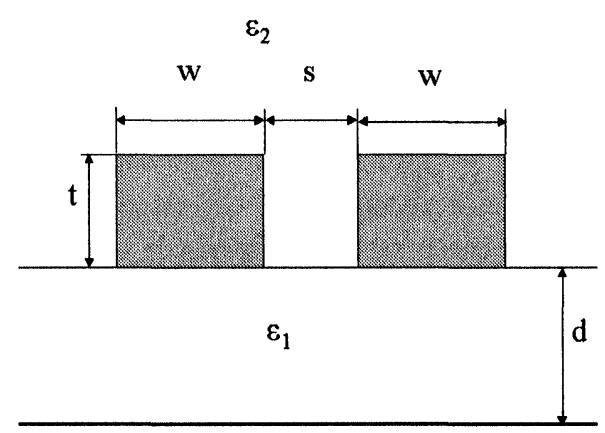

FIGURE 7 Geometry of the structure from Example 3 with coupled parallel lines $\left(\mathrm{w} / \mathrm{d}=\mathrm{w} / \mathrm{t}=3 / 1, \mathrm{~s} / \mathrm{w}=2 / 3, \varepsilon_{\mathrm{r} 1}=2\right.$ and $\left.\varepsilon_{\mathrm{r} 2}=1\right)$.

TABLE III Comparison of the numerical results for the capacitance matrix of the structure of Figure 7

\begin{tabular}{lccc}
\hline $\begin{array}{l}\text { Capacitance } \\
(p F / m)\end{array}$ & $\begin{array}{c}\text { Total charge } \\
\text { Green } \\
\text { function }[22]\end{array}$ & $\begin{array}{c}\text { Pade } \\
\text { approximation } \\
{[28]}\end{array}$ & This paper \\
\hline $\mathrm{C}_{11}$ & 93.550 & 92.644 & 93.551 \\
$\mathrm{C}_{12}$ & -8.668 & -8.305 & -8.650 \\
$\mathrm{C}_{21}$ & -8.668 & -8.305 & -8.650 \\
$\mathrm{C}_{22}$ & 93.550 & 92.644 & 93.551 \\
\hline
\end{tabular}


results are very accurate even for relatively small number of segments per conductor (only 16 per conductor).

Example 4 To show the efficiency of our new procedure, we have used it to calculate a capacitance matrix for a practical interconnect structure shown in Figure 8. It consists of three conducting lines and three dielectric layers. The numerical results for the calculated capacitance per unit length are shown in Table IV. Table IV compares numerical results calculated by the proposed technique in this paper, by the accelerating capacitance calculations using the Pade approximation [28], and the improved boundary element method [27]. The difference between our results and those of [28] is less than $3.4 \%\left(\mathrm{C}_{13}\right)$. The difference between our results and those of [27] is less than $0.6 \%$ $\left(\mathrm{C}_{11}\right.$ and $\left.\mathrm{C}_{22}\right)$. It should be noted that the accuracy of the procedure

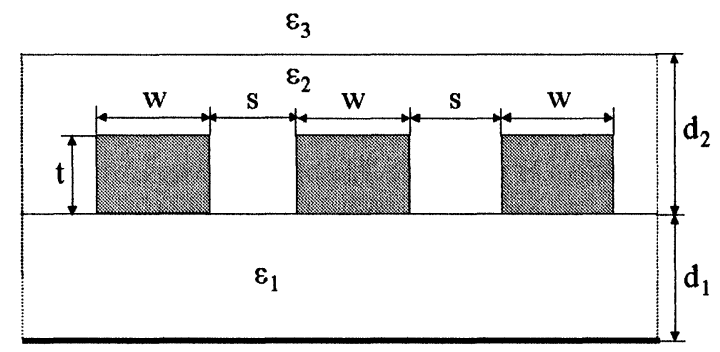

FIGURE 8 Geometry of a three line interconnect structure with three dielectric layers $\left(\mathrm{w} / \mathrm{d}_{1}=8 / 6, \mathrm{t} / \mathrm{d}_{1}=1, \mathrm{~s} / \mathrm{d}_{2}=10 / 11, \varepsilon_{\mathrm{r} 1}=9.5, \varepsilon_{\mathrm{r} 2}=4.0\right.$ and $\left.\varepsilon_{\mathrm{r} 3}=1.0\right)$.

TABLE IV Comparison of the results for the capacitance matrix of the structure of Figure 8

\begin{tabular}{lccr}
\hline $\begin{array}{l}\text { Capacitance } \\
(p F / m)\end{array}$ & $\begin{array}{c}\text { Pade } \\
\text { approximation } \\
{[28]}\end{array}$ & \multicolumn{1}{c}{$[27]$} & This paper \\
\hline $\mathbf{C}_{11}$ & 266.459 & 269.520 & 268.135 \\
$\mathbf{C}_{12}$ & -34.812 & -34.868 & -34.778 \\
$\mathbf{C}_{13}$ & -1.302 & -1.256 & -1.259 \\
$\mathbf{C}_{21}$ & -34.812 & -34.868 & -34.778 \\
$\mathbf{C}_{22}$ & 274.743 & 277.750 & 276.315 \\
$\mathbf{C}_{23}$ & -34.812 & -34.868 & -34.778 \\
$\mathbf{C}_{31}$ & -1.302 & -1.256 & -1.259 \\
$\mathbf{C}_{32}$ & -34.812 & -34.868 & -34.778 \\
$\mathbf{C}_{33}$ & 266.459 & 269.520 & 268.135 \\
\hline
\end{tabular}


can be obviously increased by increasing the number of subsections and number of terms, respectively $[14,15]$.

Example 5 In order to prove the validity of the given approach self and mutual per unit length shunt admittance (capacitance and conductance per unit length) calculated using our procedure are compared with the results of the full-wave analysis (spectral domain approach) and with those obtained by using an equivalent circuit modeling technique [29]. In Figure 9, an asymmetric coupled interconnect structure is depicted with the following electrical and geometrical parameters:

- $\mathrm{t}_{\mathrm{si}}=300 \mu \mathrm{m}, \mathrm{t}_{\mathrm{ox}}=3 \mu \mathrm{m}, \mathrm{w}_{1}=2 \mu \mathrm{m}, \mathrm{w}_{2}=1 \mu \mathrm{m}, \mathrm{T}_{1}=\mathrm{T}_{2}=1 \mu \mathrm{m}, \varepsilon_{\mathrm{si}}=$ $11.8 \mu \mathrm{m}, \rho_{\mathrm{si}}=0.01 \Omega \mathrm{cm}, \varepsilon_{\mathrm{ox}}=3.9$ and $\mathrm{s}=2 \mu \mathrm{m}$.

Figure 10a shows the variation in the distributed self and mutual capacitance per unit length $C_{11}(\omega), C_{22}(\omega)$, and $C_{12}(\omega)$, as a function of the frequency. Similarly, Figure $10 \mathrm{~b}$ shows the variation of the distributed self and mutual conductance per unit length $G_{11}(\omega), G_{12}(\omega)$, and $G_{22}(\omega)$ as a function of frequency. The solid lines are computed using the new multilayer Green's function procedure and the dashed lines are the results from the equivalent-circuit model approach [29]. It is observed that the values of the self and mutual capacitance and

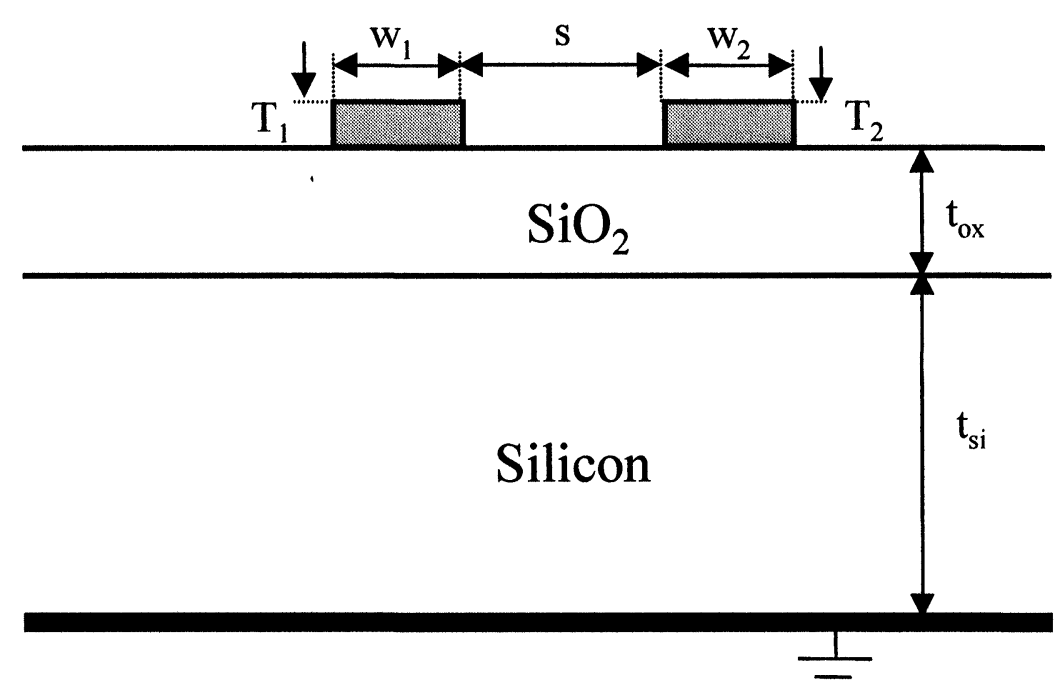

FIGURE 9 Asymmetric coupled interconnects on lossy silicon substrate. 


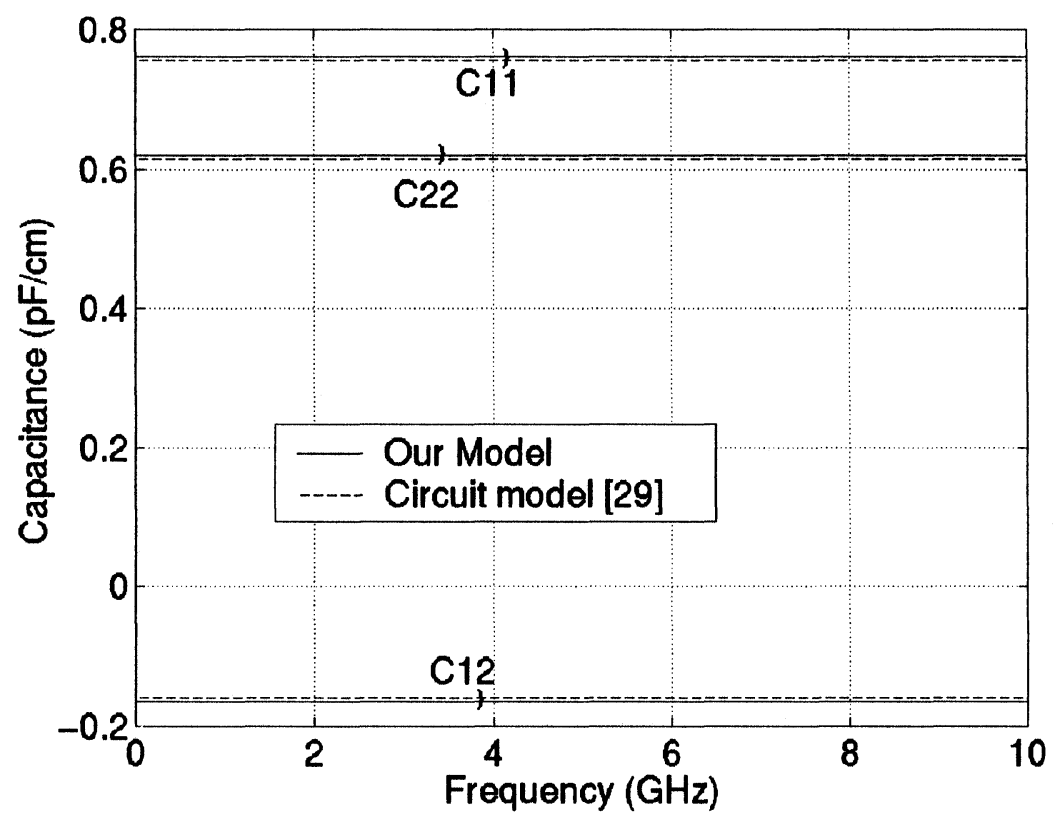

FIGURE 10a Self and mutual capacitance per unit length of asymmetric coupled interconnects on lossy silicon substrate.

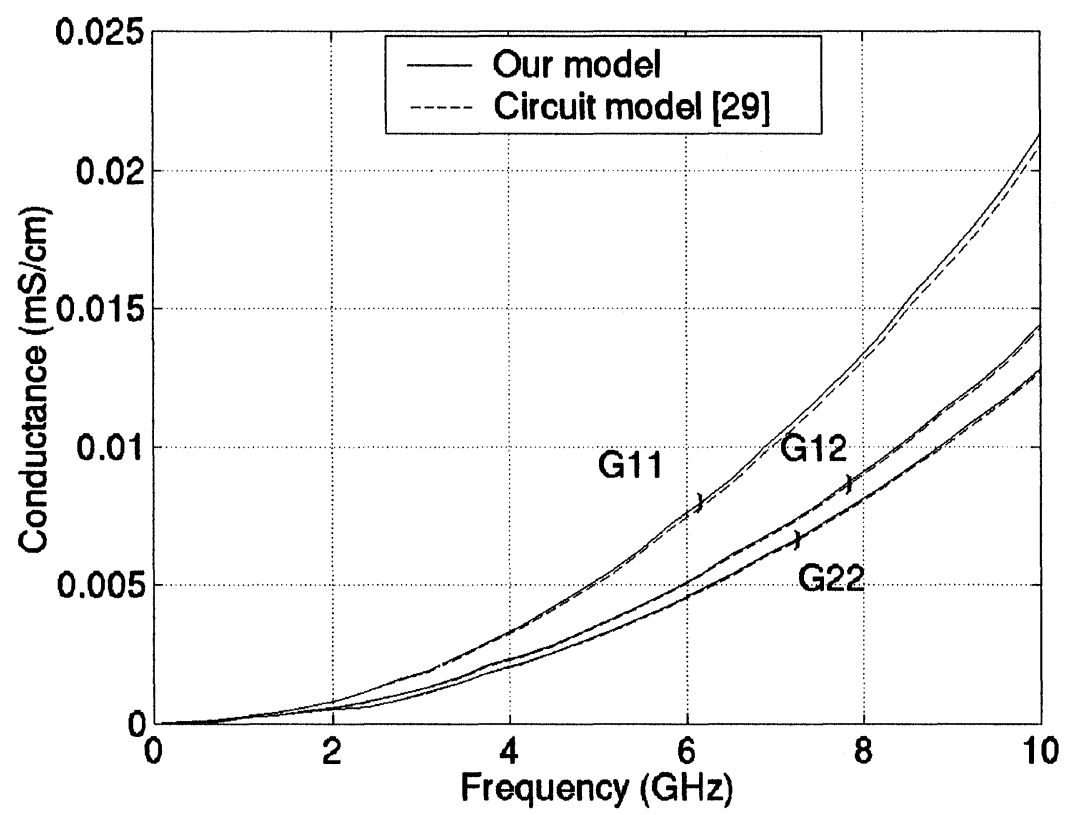

FIGURE 10b Self and mutual conductance per unit length of asymmetric coupled interconnects on lossy silicon substrate. 
conductance per unit length, respectively, are in good agreement with those of [29].

\section{CONCLUSION}

A method for modelling and simulation of interconnect networks consisting of the multi-layer lossy or semiconducting configurations with or without ground plane, a method very useful for the study of IC configurations and high speed-MCMs and other microwave and microelectronic structures, has been developed. The new expressions of the Green's functions developed in this paper are more suitable for numerical calculations of admittance per unit length (capacitance and conductance per unit length) since these expressions are designated for eliminating the singularities when the distance between the field and source point is small. The advantage of this approach is the reduced memory space needed for admittance calculations using the free-space Green's function, finite element or finite difference techniques. This is essential since the number of layers may be large for some practical designs.

A simple 2-D procedure based on the multilayer spatial Green's function has been presented for the efficient calculation of the admittance per unit length of multilayered IC interconnects taking into account the conductor transversal dimensions. The proposed method is very convenient to implement since the two-dimensional problem is reduced to a one-dimensional series for dielectric-dielectric interfaces while expressions are fully analytical in the second dimension. The procedure is very efficient because it reduces the computation cost avoiding the numerical calculation in the medium surrounding the conductors. Good accuracy has been achieved in the numerical calculations of the capacitance matrix of some 2-D interconnect configurations. The extreme convenience of the proposed technique will be highlighted when 3-D multlayer IC interconnect structures will be treated by boundary integral equation formulation and dielectric Green's function approach.

Finally, an other advantage is that the approach could be easily extended to the multilayer IC interconnects with conductors with finite/infinite thickness which can be placed everywhere in the 
structure. The method of implementation as well as example solutions are to be treated in a forthcoming papers.

\section{References}

[1] Eo, Y. and Eisenstadt, W. R., High-speed VLSI interconnect modelling based on S-parameters measurements, IEEE Trans. Comp. Hybrids, Manuf. Technol., 16, 555-562, August, 1993.

[2] Hasegawa, H., Furukawa, M. and Yanai, H. (1971). Properties of microstrip line on $\mathrm{Si}-\mathrm{SiO}_{2}$ system, IEEE Trans. Microwave Theory Tech., MTT-19, 869-881.

[3] Hasegawa, H. and Seki, S., Analysis of interconnection delay on very high-speed LSI/VLSI chips using an MIS microstrip line model, IEEE Trans. Electron Devices, ED-31, 347-352, March, 1984.

[4] Warns, C., Menzel, W. and Schumacher, H., Transmission lines and passive elements for multilayer coplanar circuits on silicon, IEEE Trans. Microwave Theory Tech., MTT-46, 616-622, May, 1998.

[5] Zaage, S. and Groteluschen, E., Characterization of the broadband transmission behaviour of interconnects on silicon substrates, IEEE Trans. Comp. Hybrids, Manuf. Technol., 16, 686-691, November, 1993.

[6] Groteluschen, E., Dutta, L. S. and Zaage, S., Quasi-analytical analysis of the broadbend properties of multiconductor transmission lines on semiconducting substrates, IEEE Trans. Comp., Pack. Manuf. Technol., Part B, 17, 376-382, August, 1994.

[7] Sorrentino, R., Leuzzi, G. and Silbermann, A., Characteristics of metal-insulatorsemiconductor coplanar waveguides for monolithic microwave circuits, IEEE Trans. Microwave Theory Tech., MTT-32, 410-416, April, 1984.

[8] Mu, T. C., Ogawa, H. and Itoh, T., Characteristics of multiconductor, asymmetric, slow-wave microstrip transmission lines, IEEE Trans. Microwave Theory Tech., MTT-34, 1471 - 1477, December, 1986.

[9] Shibata, T. and Sano, E., Characterization of MIS structure coplanar transmission lines investigation of signal propagation in integrated circuits, IEEE Trans. Microwave Theory Tech., MTT-38, 881-890, July, 1990.

[10] Mesa, F. L., Cano, G., Medina, F., Marques, R. and Horno, M., On the quasiTEM and full-wave approaches applied to coplanar multistrip on lossy dielectric layered media, IEEE Trans. Microwave Theory Tech., MTT-40, 524-531, March, 1992.

[11] Aubourg, M., Villotte, J. P., Godon, F. and Garault, Y., Finite element analysis of lossy waveguides-application of microstrip lines on semiconductor substrate, IEEE Trans. Microwave Theory Tech., MTT-31, 326-331, April, 1983.

[12] Kwon, Y. R., Hietala, V. M. and Champlin, K. S., Quasi-TEM analysis of 'slow-wave' mode propagation on coplanar microstructure MIS transmission lines, IEEE Trans. Microwave Theory Tech., MTT-35, 545-551, June, 1987.

[13] Deutsch, A., Kopcsay, G. V., Surovic, C. W., Rubin, B. J., Terman, L. M., Dunne, R. P., Gallo, T. A. and Dennard, R. H., Modelling and characterization of long onchip interconnections for high-performance microprocessors, IBM J. Res. Develop., 39, 547-567, May, 1995.

[14] Ymeri, H., Nauwelaers, B. and Maex, K., Fast and accurate analysis of multiconductor interconnects, in Proc. European Workshop Materials for Advanced Metallization (MAM 2000), Stresa, Italy, pp. 142-143, February 28-March 1, 2000.

[15] Ymeri, H., Nauwelaers, B. and Maex, K., On the mutual capacitance and inductance of a shielded interconnect four-line system, Journal of Micromechanics and Microengineering, 11, 1-4, March, 2001. 
[16] Ymeri, H., Nauwelaers, B. and Maex, K., On the modelling of multiconductor multilayer systems for interconnect applications, accepted for publication in Microelectronics Journal.

[17] Harrington, R. F. (1968). Field Computation by Moment Methods, New York: The Macmillan Company.

[18] Williams, D. F. and Marks, R. B., Accurate transmission line characterization, IEEE Microwave and Guided Wave Letters, 3, 247-249, August, 1993.

[19] Yuan, J. S., Eisenstadt, W. R. and Liou, J. J., A novel lossy and dispersive interconnect model for integrated circuit simulation, IEEE Trans. Comp. Hybrids, Manuf. Technol., 13, 275-280, June, 1990.

[20] Goossen, K. W. and Hammond, R. B., Modelling of picosecond pulse propagation in microstrip interconnections on integrated circuits, IEEE Trans. Microwave Theory Tech., MTT-37, 459-465, May, 1989.

[21] Wheeler, H. A., Formulas for the skin effect, Proc. IRE, 33, 412-424, September, 1942.

[22] Djordjevic, A. R., Bazdar, M. B., Sarkar, T. K. and Harrington, R. F. (1999). LINPAR: Matrix Parameters for Multiconductor Transmission Lines. New York: Artech House, Inc.

[23] Brews, J. R. (1986). Transmission line models for lossy waveguide interconnections in VLSI, IEEE Trans. Electron Devices, ED-33, 1356-1365.

[24] Sellberg, F., Simple determination of all capacitances for a set of parallel microstrip lines, IEEE Trans. Microwave Theory Tech., MTT-46, 195-198, April, 1998.

[25] Siegl, J., Tulaja, V. and Hoffmann, R. (1981). General analysis if interdigitated microstrip couplers, Siemens Forsch. Entwickl. Ber., 10, 228-236.

[26] Cheng, K. K. (1997). Characteristic parameters of symmetrical triple coupled CPW lines, Electron. Lett., 33, 685-687.

[27] Pan, G. W., Wan, G. and Gilbert, B. K. (1992). Edge effect enforced boundary element analysis of multilayered transmission lines, IEEE Trans. Circuits Syst. -I, 39, 955-963.

[28] Zheng, J. and Li, Z. F. (1997). Accelerating capacitance computation for interconnects in high speed MCM by Pade approximation, Electron. Lett., 33, 217-218.

[29] Zheng, J., Hahm, Y.-C., Tripathi, V. K. and Weisshaar, A., CAD-oriented equivalent-circuit modeling of on-chip interconnects on lossy silicon substrate, IEEE Trans. Microwave Theory Tech., MTT-48, 1443-1451, September, 2000. 

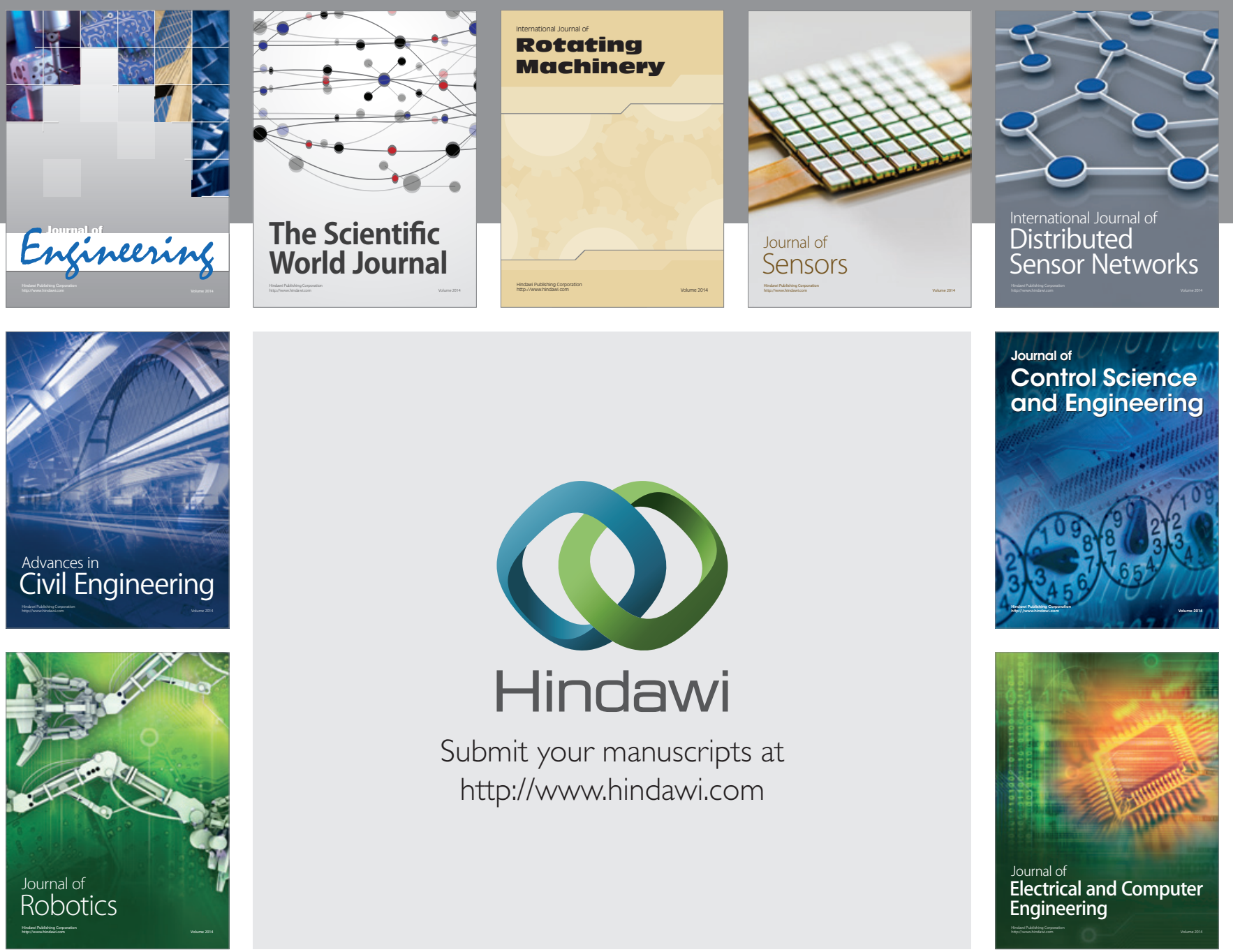

Submit your manuscripts at

http://www.hindawi.com
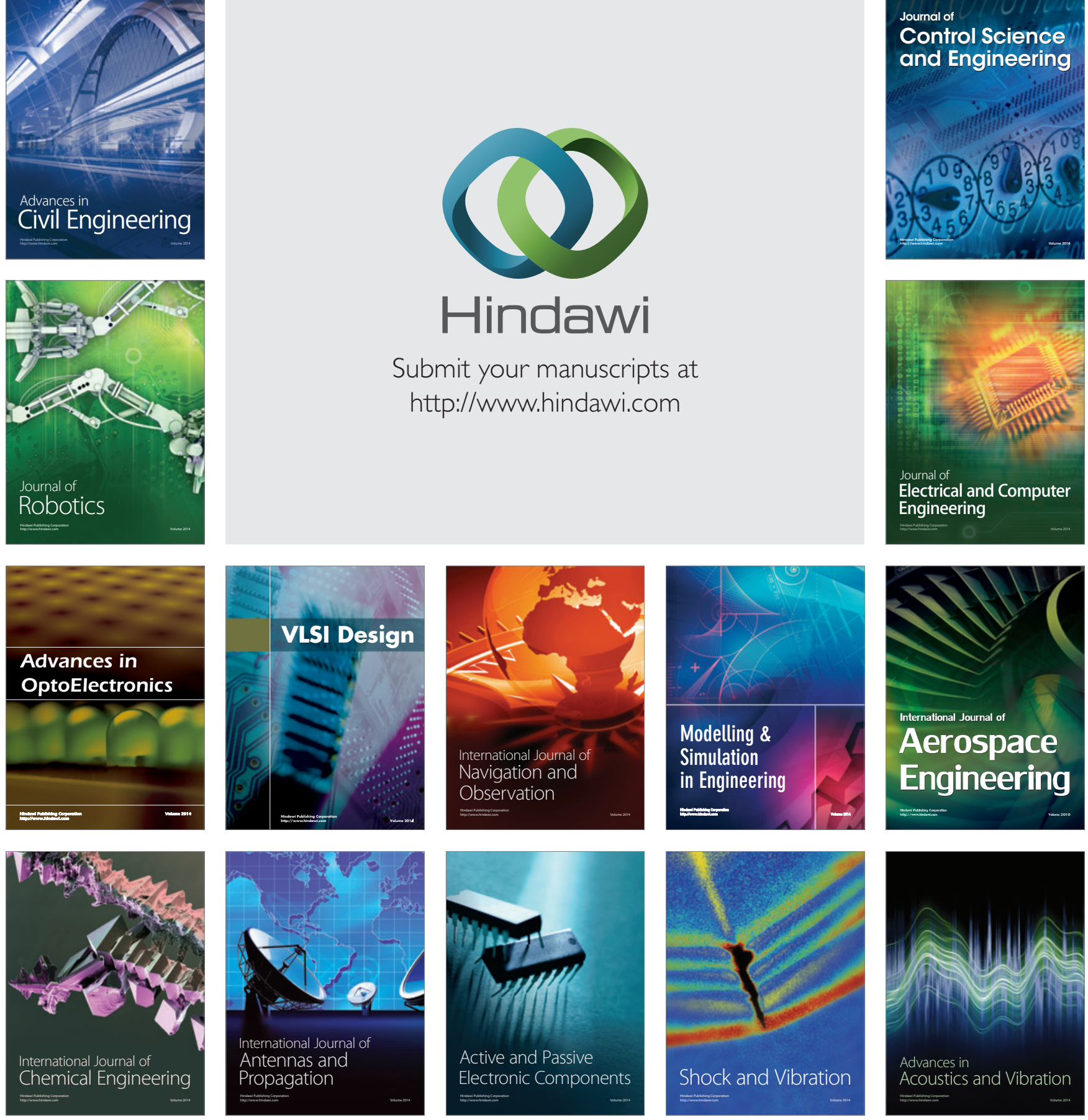